\title{
多金属氧簇与倍半硅氧烷簇构筑的簇-簇杂化分子的合成及自组装结构
}

\author{
侯占要胡敏标王维* \\ (南开大学化学学院 功能高分子材料教育部重点实验室和高分子化学研究所 合成软物质中心 天津 300071)
}

\begin{abstract}
摘要 以 Wells-Dawson 型多金属氧簇 $(\mathrm{POM})$ 与 $\mathrm{T}_{8}$ 型倍半硅氧烷簇 $(\mathrm{POSS})$ 为构筑单元, 通过点击化学反应制备了一种 由共价键连接形成的、新型的纳米簇-簇杂化分子(POM-2POSS), 并采用核磁共振(NMR)、质谱(MS)和红外光谱(IR)对 产物化学结构进行了表征. 由于两个 POSS 簇连接在 POM 簇的同一侧, 分子呈现“V”形. 同时, 利用 X 射线衍射(XRD) 及透射电子显微镜(TEM) 表征了簇-簇杂化分子在本体中通过自组装过程形成的超分子结构, 结果表明该簇-簇杂化分 子形成了有序的层状结构, 周期仅为 $5.1 \mathrm{~nm}$. 本研究获得结果对以这类纳米簇为构筑单元构筑新型杂化分子以及通过 自组装过程形成的、有序超分子结构的新型杂化材料的设计及制备提供了一个新的思路.
\end{abstract}

关键词 杂化分子; 多金属氧簇; 倍半硅氧烷簇; 点击化学; 自组装

\section{Synthesis and Self-Assembled Structure of A Cluster-Cluster Hybrid Molecule Composed of POM and POSS Clusters}

\author{
Hou, Zhanyao $\mathrm{Hu}$, Minbiao $\quad$ Wang, Wei* \\ (Center for Synthetic Soft Materials, The Key Laboratory of Functional Polymer Materials of Ministry of Education and \\ Institute of Polymer Chemistry, Nankai University, Tianjin 300071)
}

\begin{abstract}
Polyhedral oligosilsesquioxane (POSS) and polyoxometalate (POM) are two kinds of clusters having totally different physical and chemical properties. For instance, the POSS cluster dissolves in weakly polar solvents, such as toluene, while the POM cluster, encapsulated by tetrabutylammonium counterions, dissolves in strongly polar solvents, such as acetonitrile, meaning the strong incompatibility. Based on this reason and their fixed shape, a novel cluster-cluster hybrid molecule with a V-shaped molecular structure (POM-2POSS) was rationally designed by covalently linking the two POSS clusters on the one side of the POM cluster. In the experiment, a two-azido-containing organosilyl derivative of a Wells-Dawson-type POM cluster and a one-propargyl-containing derivative of a POSS cluster were prepared at first. Then, the cluster-cluster hybrid was successfully synthesized by $\mathrm{Cu}$-catalyzed click reaction between the two azide groups in the one POM derivative and the two propargyl groups in the two POSS derivatives. The chemical structure of this hybrid molecule was carefully characterized by NMR, ESI-MS and IR. In view of their strong incompatibility and of the particular three-dimensional (3D) structure POM-2POSS was expected to be able to self-assemble into ordered supramolecular structures. In the sample preparation POM-2POSS was dissolved in acetonitrile with a concentration of $5 \mathrm{mg} / \mathrm{mL}$, and then the solution was dropped onto silicon substrates to prepare the thin film samples, finally the thin film samples were annealed in an acetonitrile vapor for 14 d. The film on the silicon substrate was characterized by XRD. The thin film samples for TEM characterization were made at first by floating onto the water surface and then transferred onto copper mesh. The structural analyses clearly demonstrated that the hybrid molecule self-assembled into a highly ordered lamellar morphology with a $5.1 \mathrm{~nm}$ periodicity, smaller than those found in block copolymers with a similar molecular weight. Formation of the highly ordered morphology reflects a self-assembly process due to absence of intermolecular entanglements, while the sub-5 nm periodicity is because of the 3D structures of the two building blocks. The findings provide a new platform for understanding of the self-assembly of nano-clusters and for development of novel hybrid materials.

Keywords hybrids; polyoxometalates; polyhedral oligosilsesquioxanes; click reaction; self-assembly
\end{abstract}

\section{1 引言}

在材料学科中, 研究和发展新型杂化材料(或者纳 米复合材料)已成为最吸引科学家和工程师们关注的一 个研究领域, 其主要原因是某一种甚至某一类传统材料
(比如金属、陶瓷和塑料等)已不能满足现代科学技术对 材料功能方面提出的新需求, 而通过杂化的方式制备的 杂化材料，能够将不同传统材料的优异性能相互结合， 展现出诱人的、可以满足新需求的功能. 近数十年的研 究表明，成功制备这样的功能材料的关键在于在分子或

\footnotetext{
*E-mail: weiwang@nankai.edu.cn

Received August 6, 2013; published August 28, 2013.
}

Supporting information for this article is available free of charge via the Internet at http://sioc-journal.cn.

Project supported by the National Natural Science Foundation of China (Nos. 21274069 and 21334003) and Open Research Fund of State Key Laboratory of Polymer Physics and Chemistry, Changchun Institute of Applied Chemistry.

项目受国家自然科学基金(Nos. 21274069 和 21334003)与高分子物理与化学国家重点实验室开放研究基金资助. 
者纳米级将两类不同的材料进行杂化，进而控制大尺度 的超分子结构, 实现杂化材料功能的最优化 ${ }^{[1 \sim 4]}$. 为此, 在纳米杂化材料研究领域里, 科学家们越来越感兴趣的 课题是, 通过合理的分子设计, 将两类性能和功能各异 的构筑单元在分子级或者纳米级通过共价键连接, 构成 新型的杂化分子或者大分子, 进而在纳米级以上的空间 尺度上调控由自组装过程形成的超分子结构, 目的在于 优化甚至最大化杂化材料的性能和功能, 为开发新型的 功能器件奠定基础. 由此可见, 功能杂化材料的研究充 分地体现了纳米科学研究的 “自下而上” 的精髓 ${ }^{[14]}$.

最近的研究表明, 具有特殊拓扑结构的构筑单元形 成的杂化分子可能展现出奇特的超分子组装结构 $\left.{ }^{[5} 8\right]$. 多金属氧簇 $(\mathrm{POMs})^{[9 \sim 15]}$ 、倍半硅氧烷簇 $(\mathrm{POSS} S)^{[16,17]}$ 和 富勒烯 $(\mathrm{C} 60)^{[18,19]}$ 等是业已使用的、具有特殊拓扑结构的

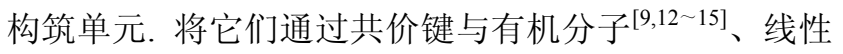
聚合物 ${ }^{[10,11]}$ 、寡聚物 ${ }^{[16]}$ 等结合制备了一系列有趣的称为 形状两亲性分子(Shape Amphiphiles). 很明显两个构筑 单元在拓扑结构上存在明显的差异. 目前, 这类杂化两 亲性分子的自组装行为正在成为纳米科学中一个非常 重要的研究课题.

多金属氧簇(有时也称为多酸)是由前过渡金属元素 的最高价氧化态通过氧连接而成的一类具有明确结构 的多阴离子簇 ${ }^{[20,21]}$. 它的结构、拓扑形状及组成元素的 多样性赋予其在许多领域具有重要的应用价值. 最近二 十年里, 发展了一系列的 POMs 有机功能化的方法, 创 造出数目巨大的、含 POM 族的有机-无机杂化分子, 更 加可喜的是它们可能兼有有机构筑单元和 POM 的功能, 是发展含 POMs 功能材料候选者 ${ }^{[22 ~ 25]}$. $\mathrm{T}_{8}$ 型 POSS 簇的 内核具有类似二氧化硅结构的硅氧六面体, 外壳是通过 硅原子连接的有机取代基, 其分子尺寸及性质随有机取 代基的不同而变化, 是目前研究最多、结构规整和确定 的族合物之一. 在杂化材料研究中, 它常与聚合物及其 它有机物进行杂化，制备 POSS 基的功能材料 ${ }^{[26,27]}$.

最近几年里, 我们研究组正在利用具有结构和功能 都有多样性的 POMs 和 POSSs 的分子簇作为结构单元, 构筑尺寸在纳米级的新型纳米簇-族杂化分子. 在一个 已经发表的工作中, 我们报道了将一个三个钒原子取代 的 Wells-Dawson 型 POM 与一个 POSS 通过共价键连接 在一个有机小分子链的两端, 形成了亚铃型杂化分子 (POM-organic-POSS), 发现组装后得到了长周期小于 5 $\mathrm{nm}$ 的层状微相分离结构 ${ }^{[14]}$. 为了弄清分子拓扑形状与 超分子组装结构的关系, 本研究工作进一步设计并合成 了一种 “V” 形分子 POM-2POSS, 即在一个 POM 簇的 一侧连接上两个 POSS 族. 由于簇-族杂化分子中 POM 段与 POSS 段的不相容, 在本体样品中我们发现了由两 类分子簇交替排列形成的层状结构, 长周期为 $5.10 \mathrm{~nm}$. 这样小的长周期源自于我们使用了两种三维的纳米簇 来构筑族一簇杂化分子. 我们获得的研究结果对随后的
设计和合成纳米簇-族杂化分子进而操控通过组装形成 的多级超分子结构提供了新的途径.

\section{2 结果与讨论}

\section{1 “V” 形簇一簇杂化分子 POM-2POSS 的分子设计 与合成}

选用 Wells-Dawson 型 POM 簇和 $\mathrm{T}_{8}$ 型 POSS 族作 为目标簇一族杂化分子的构筑单元. 多金属氧簇 $\mathrm{K}_{10}\left[\alpha_{2}-\mathrm{P}_{2} \mathrm{~W}_{17} \mathrm{O}_{61}\right] \cdot 10 \mathrm{H}_{2} \mathrm{O}$ 为带有一个缺位的铇多酸, 中 心杂原子为两个磷原子，带六个负电荷. 该阴离子簇的 外围抗衡离子可以改变, 当抗衡离子为有机阳离子时可 溶于极性较大的有机溶剂，例如二甲基甲酰胺(DMF), 便于对该无机阴离子进行有机修饰. 若无特殊说明, 本 工作中使用的多金属氧簇外围抗衡离子均为四丁基铵 阳离子 $\left(\mathrm{Bu}_{4} \mathrm{~N}^{+}\right)$. 在多金属氧族的缺位处, 可以与硅氧 烷通过硅氧化反应进行有机修饰. 本工作参考文献 ${ }^{[28]}$ 将两个叠氮基团修饰在多金属氧族的一端，命名为 $\mathrm{POM}-2 \mathrm{~N}_{3}$. 使用的倍半硅氧烷分子簇是 1 -氨基丙 基-3,5,7,9,11,13,15-七异丁基倍半硅氧烷, 即倍半硅氧 烷分子簇外围含有七个异丁基和一个氨基丙基取代基. 本工作中戊炔酸与氨基进行酰胺化反应将炔基修饰在 倍半硅氧烷簇上, 命名为 POSS-yne. 将 POM- $2 \mathrm{~N}_{3}$ 上的 两个叠氮和两个 POSS-yne 上的炔基通过点击化学反应 即可得到目标分子: “V”形簇-簇杂化分子 POM-2POSS.

“V” 形簇-族杂化分子 POM-2POSS 的合成路线如 图 1 所示. POSS 族由商业化的氨基倍半硅氧烷衍生物 (1: $\left.\mathrm{POSS}-\mathrm{NH}_{2}\right)$ 为初始原料, 其中氨基与戊炔酸在 EDC/DMAP 的活化作用下经酰胺化反应在 POSS 族的 一臂上引入了炔基, 得到带炔基的倍半硅氧烷族 $(2$ : POSS-yne). 根据文献 ${ }^{[29]}$ 合成的 POM 簇(3)在缺位处与 氯甲基苯基三甲氧基硅烷进行硅氧化反应引入两个氯 基团, 再使用 $\mathrm{NaN}_{3}$ 将氯取代为叠氮基团得到叠氮修饰 的多金属氧簇( 4: POM- $2 \mathrm{~N}_{3}$ ). POSS-yne 和 POM- $2 \mathrm{~N}_{3}$ 在 $\mathrm{DMF}$ 溶剂中以 $\mathrm{CuSO}_{4} \cdot 5 \mathrm{H}_{2} \mathrm{O}$ 和抗坏血酸为催化剂 ${ }^{[30]}, 50$ ${ }^{\circ} \mathrm{C}$ 下反应 $5 \mathrm{~d}$ 即可得到目标簇一族杂化分子(5: POM2POSS).

使用核磁共振 $\left({ }^{1} \mathrm{H}\right.$ NMR, ${ }^{13} \mathrm{C}$ NMR, ${ }^{31} \mathrm{P} \mathrm{NMR}$ 和 ${ }^{29} \mathrm{Si}$ NMR)、质谱(ESI-MS)、红外(IR)及元素分析确定了每一 步反应产生的化合物和最终族-簇杂化分子的结构, 详 细表征数据见支持信息. 化合物 $\mathbf{2}$ 和 $\mathbf{4}$ 较易制备及提纯， 利用点击化学反应将 2 和 4 连接成 POM-2POSS (5) 是合 成关键步骤，本文将重点讨论.

族-簇杂化分子 POM-2POSS 的 ${ }^{1} \mathrm{H}$ NMR 数据如图 $2 \mathrm{~A}$ 所示, 各个峰都能够得到正确的归属(详细的归属见 实验部分). 炔基与叠氮基团经点击化学反应所生成的 三唑五元环体现在核磁数据上是 $\mathrm{k}$ 峰的出现. 根据 $\mathrm{k}$ 峰 与 $\mathrm{h}$ 及 $\mathrm{i}$ 峰的面积积分比值为 $2: 4: 4$ 可以充分证明化 
<smiles></smiles>

1: POSS-NH

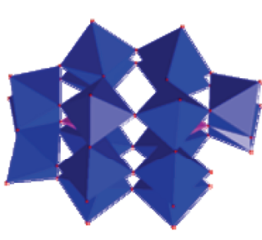

3: POM

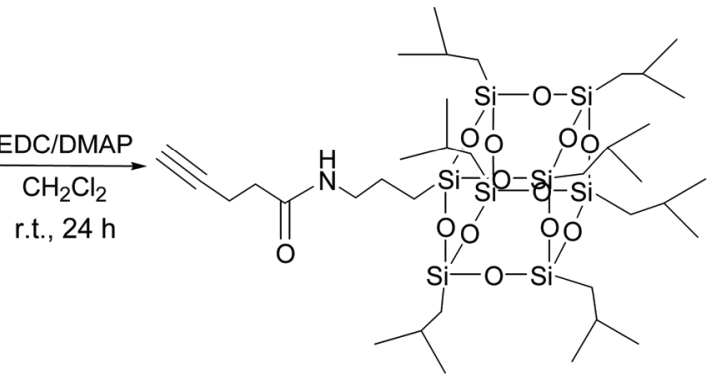

$\mathrm{CuSO}_{4} \cdot 5 \mathrm{H}_{2} \mathrm{O}$ Ascorbic Acid DMF, $50{ }^{\circ} \mathrm{C}, 5 \mathrm{~d}$
2: POSS-yne

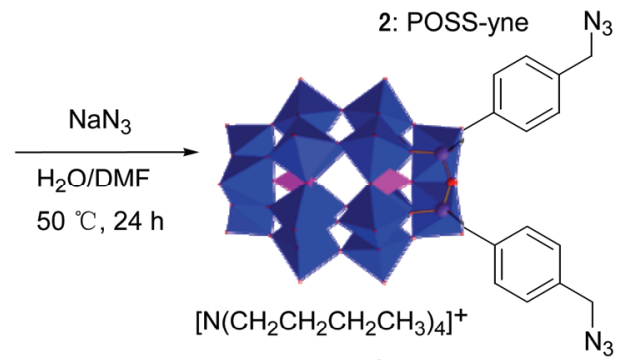

4: $\mathrm{POM}-2 \mathrm{~N}_{3}$

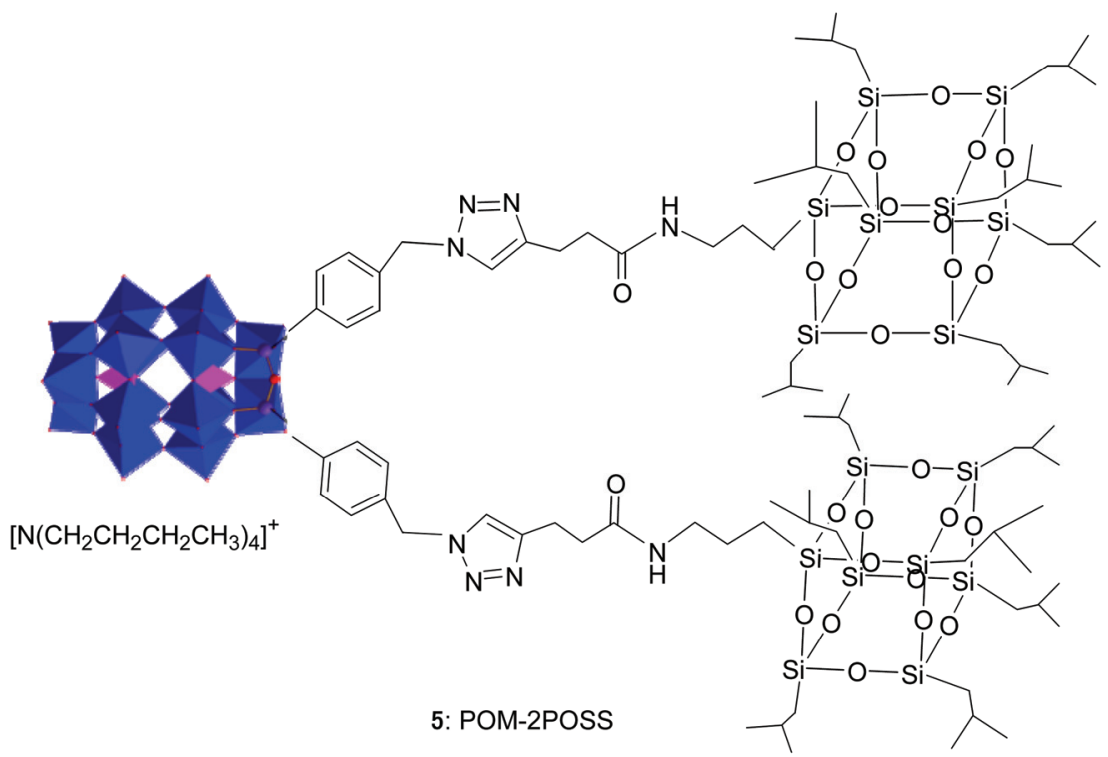

图 1 “V”形簇-簇杂化分子 POM-2POSS 的合成路线

Figure 1 Synthetic route to the POM-2POSS hybrid molecule

合物 $\mathbf{2}$ 与 $\mathbf{4}$ 的反应等物质的量比为 $2: 1$, 即得到了目标 簇-簇杂化分子 POM-2POSS. 由于在有机改性中 POM 簇通常不太稳定, 利用 ${ }^{31} \mathrm{P} N \mathrm{NMR}$ 表征方法证实了在合成 过程中这个 $\mathrm{POM}$ 簇并没有解体. 图 2B 所示了点击反应 前后 POM 簇中两个 $\mathrm{P}$ 的化学位移都没有发生变化. 实 验上遇到的问题是多阴离子的 POM 簇可能吸附催化剂 阳离子, 从而增大了提纯目标产物的难度. 图 $2 \mathrm{C}$ 为簇簇杂化分子 EDX 表征数据, 谱图中没有铜元素的信号, 表明所得样品没有催化剂铜的残留.

\section{2 杂化分子自组装行为研究}

\subsubsection{POM-2POSS 的物理化学性质的差异}

使用的 Wells-Dawson 型 POM 簇分子为椭球形, 长
轴为 $1.2 \mathrm{~nm}$, 短轴为 $1.0 \mathrm{~nm}$; POSS 簇为六面体状, 对角 线长度为 $1.3 \mathrm{~nm}$; 连接分子簇之间的有机分子链在 POM 的长轴方向有效长度约 $0.7 \mathrm{~nm}$; POM-2POSS 分子 长度为 $3.3 \mathrm{~nm}$. 在物理化学性质上的差异之一表现在不 同的溶解性上，这就赋予了簇-簇杂化分子中的 POM 与 POSS 段的不相容性. 这个 POM 簇被六个 $\mathrm{Bu}_{4} \mathrm{~N}^{+}$抗衡阳 离子包围. 这种盐形式的 POM 簇能溶解在极性较大的 有机溶剂中, 常见的良溶剂为乙腈 $\left(\mathrm{CH}_{3} \mathrm{CN}\right)$ 和 DMF. 这 个 POSS 簇外围有七个异丁基取代基, 它的良溶剂则是 极性较小的有机溶剂, 如甲苯和二氯甲烷等. 显然, 这 个簇-簇杂化分子中两种构筑单元的不相容性，类似于 两亲性嵌段共聚物所具有的性质, 应该能发生微相分离 现象而形成有序的超分子结构. 簇-簇杂化分子的分子 

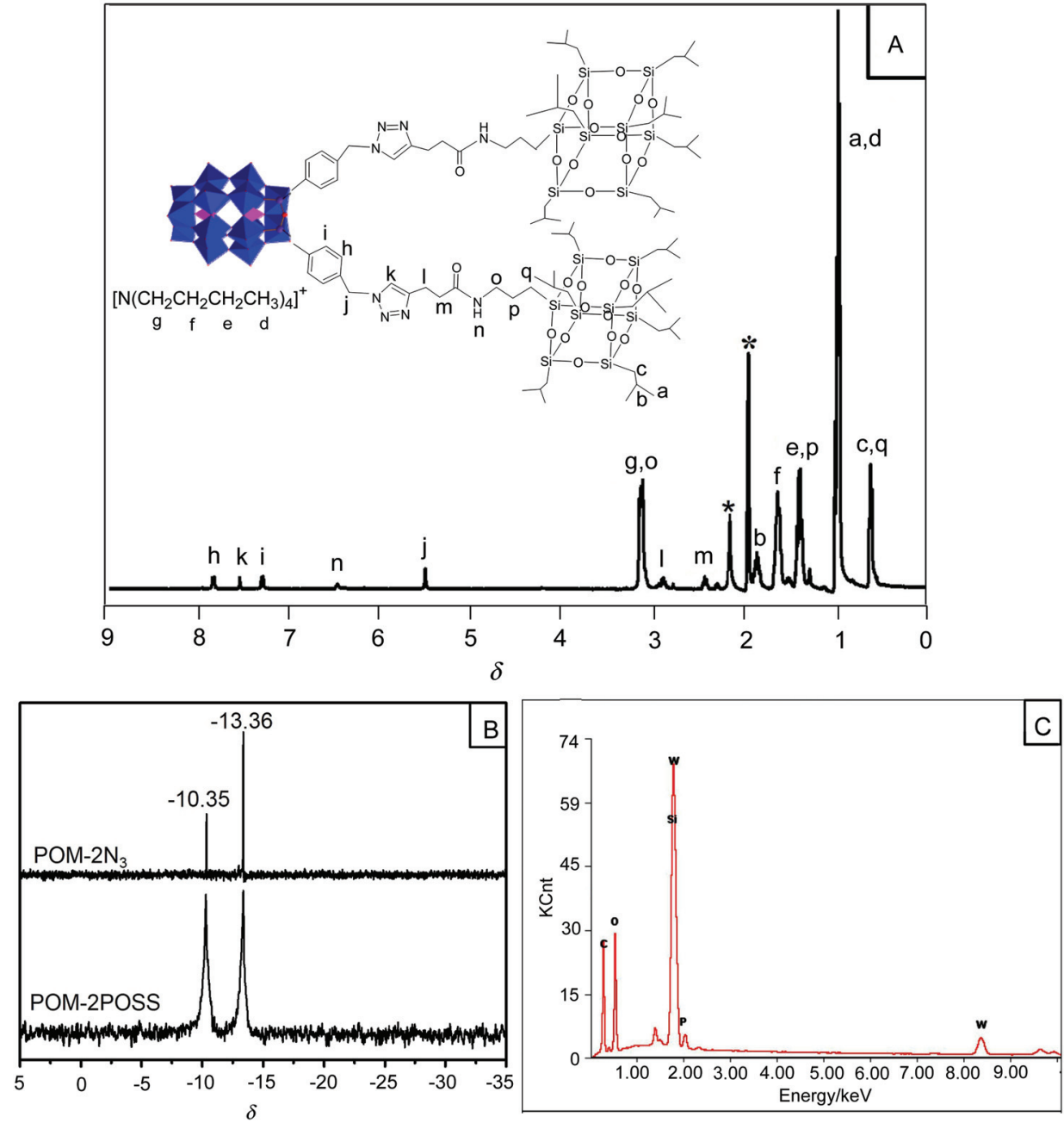

图 2 (A) 簇-簇杂化分子 POM-2POSS 的 ${ }^{1} \mathrm{H}$ NMR 谱图. (B) POM- $2 \mathrm{~N}_{3}$ 和 POM-2POSS 的 ${ }^{31} \mathrm{P}$ NMR 谱图. (C) POM-2POSS 的 EDX 谱图 Figure 2 (A) ${ }^{1} \mathrm{H}$ NMR spectrum of the POM-2POSS hybrid. (B) ${ }^{31} \mathrm{P}$ NMR spectra of POM- $2 \mathrm{~N}_{3}$ and POM-2POSS. (C) EDX spectrum of the POM-2POSS hybrid molecule

量为 $7860 \mathrm{Da}$, 分子量接近在超分子自组装研究中常使 用某些嵌段聚合物的分子量. 然而，该分子是由两个 POSS 和一个 POM 纳米簇构成, 会具有不同于常见的嵌 段共聚物的物理特征，比如分子尺寸仅为 $3.3 \mathrm{~nm}$, 远远 小于嵌段共聚物, 且簇与簇间无缠结, 为此形成的微相 分离结构特征和尺度可能不同于传统嵌段共聚形成的 结构.

\subsection{2 超分子结构的 XRD 表征和研究}

为了使用 $X$ 射线粉末衍射 $(X R D)$ 方法研究簇-簇杂 化分子在本体中的超分子结构, 我们将簇-簇杂化分子 的乙腈溶剂浇铸在硅片上形成膜样品, 然后在乙腈的饱 和蒸汽中退火 $14 \mathrm{~d}$. 所得到膜样品的 XRD 表征结果如 图 3 所示, 显示衍射强度 $I$ 与散射角 $2 \theta$ 的作图, 可以清 楚地看见在 $2 \theta=1.73^{\circ}$ 有一个非常强的衍射峰, 在实验 条件下强度高达 40000 . 同时, 在插图中我们还能看见 在 $2^{\circ} \leqslant 2 \theta \leqslant 10^{\circ}$ 的区域里, 有三个非常弱的峰, 出现在 $2 \theta=3.44^{\circ}, 5.08^{\circ}$ 和 $6.82^{\circ}$, 强度都低于 600 . 由 Bragg 方

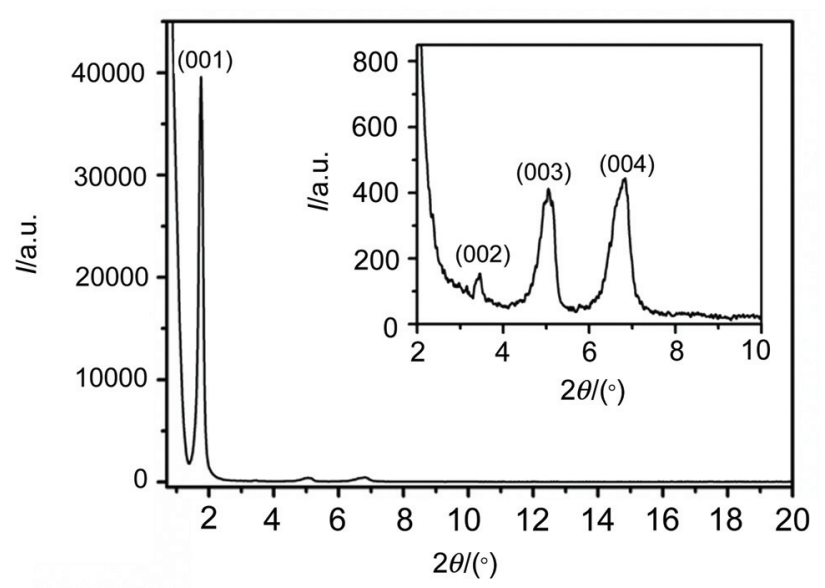

图 3 簇-族杂化分子 POM-2POSS 的 XRD 表征结果

Figure 3 XRD pattern of the POM-2POSS hybrid film sample

程可以计算各个衍射峰所对应长周期 $d=5.10,2.57$, 1.74 和 $1.30 \mathrm{~nm}$. 我们发现四个衍射峰所对应 $d$ 值的倒数 比值为 $1: 2: 3: 4$, 即 $1 / d_{1}: 1 / d_{2}: 1 / d_{3}: 1 / d_{4}=1: 2:$ 
3:4, 说明簇-族杂化分子 POM-2POSS 形成了典型的 层状结构, 其长周期为 $d_{1}=5.10 \mathrm{~nm}$. 四个散射峰对应的 Miller 指数(001), (002), (003)和(004)已经在图 3 中注明. 与此同时, 我们采用 Scherrer 方法分析了沿着垂直于 (001)面方向上, 层状结构的有效相关长度为 $33.5 \mathrm{~nm}$. 由于有效相关长度与长周期比值约为 6.6 , 也就是说, 在垂直于 $(001)$ 面方向上, 统计意义上有六条片层完全 平行地排列. 这些 XRD 表征和分析结果都说明, 通过 溶剂退火方法在硅片上铸膜中簇一簇杂化分子形成了高 度有序的超分子结构。

\subsection{3 超分子结构 TEM 表征和研究}

为了更加直观地观测杂化分子所形成的超分子结 构, 我们对铸膜样品进行了场发射透射电子显微镜的表 征. 实验中, 将预注在硅片上所形成的薄膜样品, 转移 到稀 HF 水面上, 再使用铜网将漂浮在水面上的超薄样 品捞起, 并进行了透射电子电镜(TEM)表征.

图 4 为族一簇杂化分子的明场 TEM 照片, 清楚地显 示出层状周期结构特征. 由于簇-族杂化分子含有十七 个铇的 POM 族比含八个硅的 POSS 具有强的电子散射 能力, 在样品无需染色的条件下就可直接在 TEM 下观

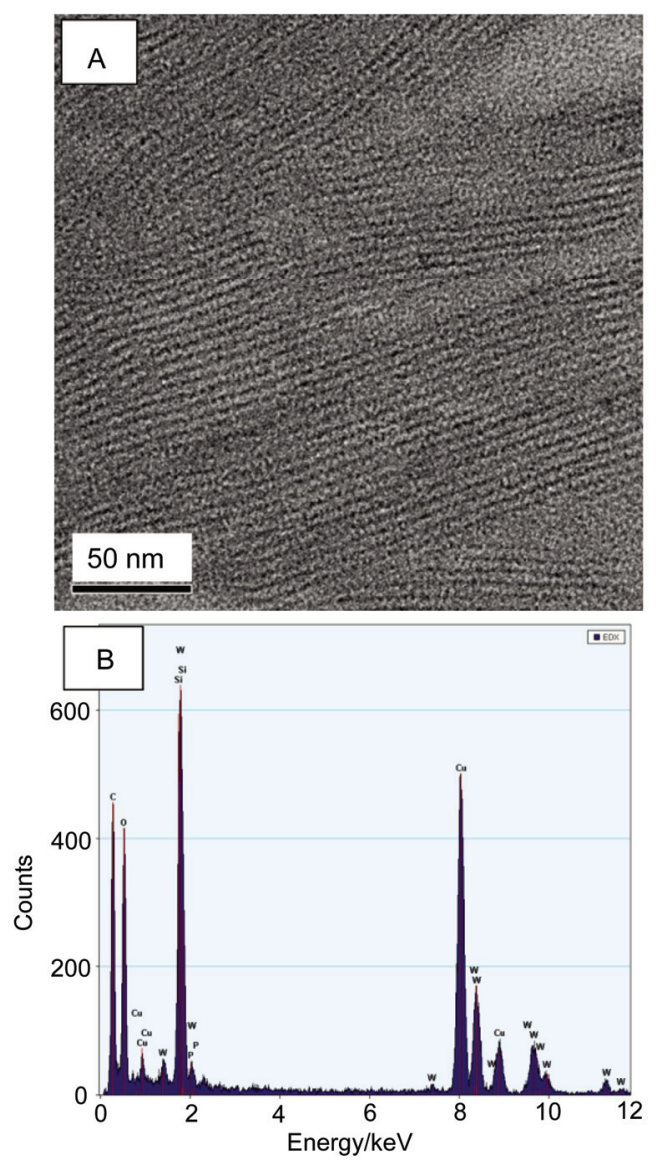

图 4 簇-簇杂化分子 POM-2POSS 膜样品的 TEM 表征照片(A)和 EDX 分析结果(B)

Figure 4 TEM image (A) and EDX pattern (B) of the ultrathin film of the POM-2POSS hybrid
察到了族-簇杂化分子形成的结构，有明暗条带结构交 替排列而形成. 暗的区域代表 POM 的富集区，而较亮 区域代表 POSS 富集区域. 其次，对样品进行了电子能 谱(EDX)的分析，结果见插图，POM 段及 POSS 段所含 有的特征元素 W, P, Si 都能被检测出来, 说明了我们所 观测到的层状结构是由杂化分子形成的.

我们还对 TEM 照片进行了详细的分析处理, 通过 Fourier 及反 Fourier 变换的处理, 可以除去 TEM 照片中 的高频噪音, 从而在不改变照片真实性的情况下使照片 更加清晰. 图 5 选自图 4 部分区域, 经过 Fourier 及反 Fourier 变换处理并放大后的 TEM 图, 可以发现层状周 期结构更加明显. 进一步对层状尺寸的统计分析表明其 周期大小为 $5.1 \mathrm{~nm}$, 这与 XRD 测定的周期值非常吻合. 其次, 从图 5 中我们可以看出, 同层纳米线是不连续的, 说明我们设想纳米线是由刚性分子簇相互堆积而形成 的是合理的. 簇一族杂化分子中, 刚性纳米簇的堆积不 同于嵌段共聚物，没有分子链间的相互缠结，从而堆积 结构是不连续的.

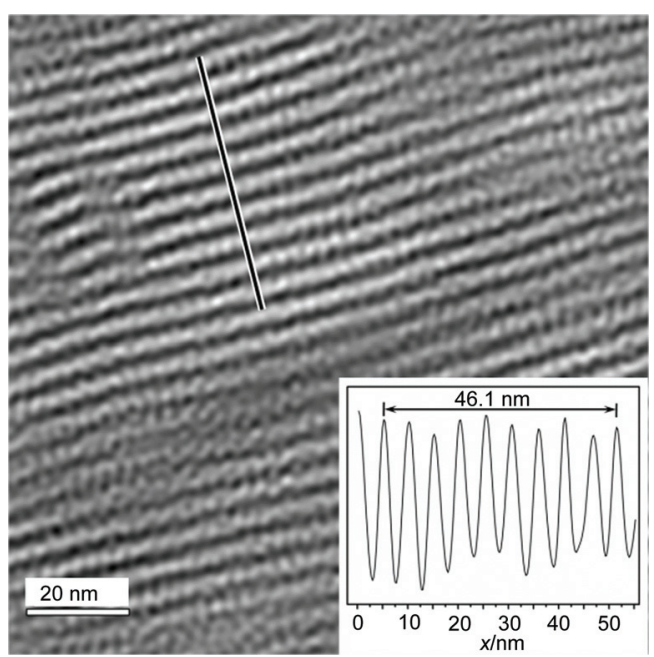

图 5 图 4 中 $\mathrm{TEM}$ 照片经放大且. Fourier 及反 Fourier 变换处理后的 TEM图. 图中的黑线为测定层状结构时扫描区域. 插图为层状周期结 构统计分析结果

Figure 5 Enlarged image of the TEM image in Figure 4 after processed by FFT and I-FFT analysis. Inset shows a statistical analysis of lamellar thickness

\subsection{4 建议的超分子结构的形成过程和堆积模型}

为了有利于讨论超分子结构的形成过程和簇一簇杂 化分子在超分子结构中的堆积模式，有必要再次介绍 POM 簇和 POSS 族的分子特征尺寸及性质. POM 族为椭 球形, 长轴为 $1.2 \mathrm{~nm}$, 短轴为 $1.0 \mathrm{~nm}$; POSS 族为六面体 状, 对角线长度为 $1.3 \mathrm{~nm}$; 连接簇之间的有机分子链的 有效长度约 $0.7 \mathrm{~nm}$; 因而估计 POM-2POSS 分子长度为 $3.3 \mathrm{~nm}$. 更加重要的是, POM 簇溶解在强极性溶剂中, 而 POSS 簇溶解在非极性溶剂中, 表明它们高度互不相 容，但被有机链以共价键方式连接起来. 对于这样的 簇一族杂化分子, XRD 和 TEM 表征和数据分析均表明, 在样品制备过程中，当溶剂逐渐地挥发时簇一族杂化分 
子组装成高度有序的层状结构, 其周期大小约为 5.1 $\mathrm{nm}$.

在此, 我们首先推演在从稀溶液转变为本体样品的 过程中, 族-簇杂化分子组装成有序的层状结构的过程. 可以推断, 在稀溶液里, 簇-簇杂化分子被溶剂分子包 围着见图 6A. 假设在一个特定的临界浓度时, 族一簇杂 化分子开始接触. 由于 POM 簇段和 POSS 族段互不相 容, 就开始相互排斥, 导致不同簇一簇杂化分子中的 POM 族段选择性地聚集在一起, 而 POSS 族也选择性地 聚集在一起, 发生了相分离. 然而, POM 簇段和 POSS 簇段间是共价键, 相分离的长度只能在一个与分子尺度 相当的微观尺度范围内, 即只发生了微相分离, 见图 6B. 为此, 簇-簇杂化分子形成的微相分离结构的尺度 在分子尺度范围内.
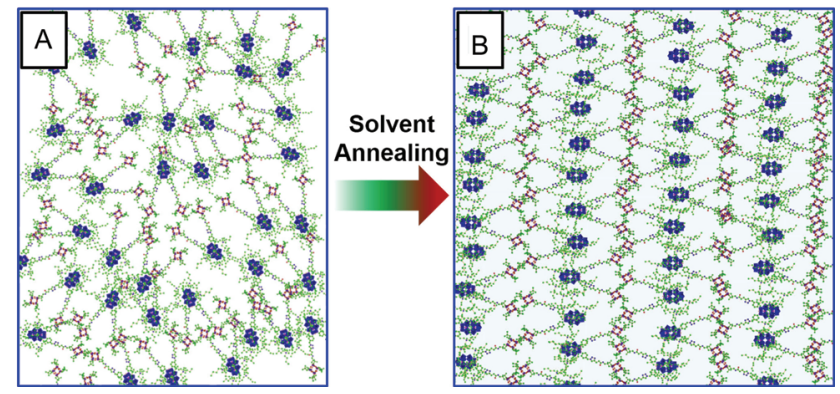

图 6 建议的超分子结构的形成过程和簇一簇杂化分子的堆积模型

Figure 6 Suggested formation of supramolecular structure and packing model of cluster-cluster hybrids in supramolecular structure

另外一个重要的问题是在超分子结构里簇-簇杂化 分子的堆积模型. 前人对嵌段共聚物的系统研究已经表 明, 形成的超分子结构取决于两段的相对体积分数. 在 两段的体积分数之比接近 1 时, 通常形成层状超分子结 构 ${ }^{[31]}$. 我们的 XRD 和 TEM 表征和数据分析均表明, 簇簇杂化分子组装成层状结构, 说明 POM 簇段和 POSS 簇段的体积接近相等. 再注意 “ $\mathrm{V}$ ” 形簇-簇杂化分子由 一个 POM族与两个POSS 簇在 POM 的同一侧连接而成. 也就是说, 两个 POSS 簇的体积约等于一个 $\mathrm{POM}$ 簇的体 积. 鉴于上面的讨论, 我们提出了图 $6 \mathrm{~B}$ 示意簇-簇杂化 分子 POM-2POSS 构筑的超分子组装结构的堆积模型. 在这个模型中, 层状结构的周期为 $5.1 \mathrm{~nm}$, 大于簇 - 簇 杂化分子的长度 $(3.3 \mathrm{~nm})$, 但是小于分子长度的 2 倍 $(6.6$ $\mathrm{nm})$, 这也预示着簇-簇杂化分子并非排列成完美的 “头 -头” 形式, 而是在 POM 簇层中 POM 簇相互间有 “之” 字形的排列, POSS 簇也是如此. 这就导致一个长周期介 于一倍和二倍分子长度之间的层状结构. 但是, 这不意 味着, 层状结构并不完美. 事实上, XRD 表征结果表明, 与我们知道的嵌段共聚物的 XRD 表征结果相比, (001) 散射峰的强度非常强, 半峰宽也窄, 预示着高度有序的 结构. 究其原因, 相信是与 POM 簇和 POSS 族的几何特 征和无缠结的特性密切相关.

\section{3 结论}

本文成功的以 Wells-Dawson 型多金属氧族(POM) 及 $\mathrm{T}_{8}$ 型倍半硅氧烷簇(POSS)为原料, 通过点击化学方 法合成了一种具有 “ $V$ ” 字形的无机族与族的杂化分子 POM-2POSS. 超分子结构研究表明簇一簇杂化分子经组 装后形成了长周期为 $5.1 \mathrm{~nm}$ 的有序层状结构. 进一步的 分析表明形成层状结构是由于 POM 簇段及 POSS 族段 的不相容性, 即两段簇合物的物理化学性质巨大差异驱 动两段发生微相分离所致. 而长周期为 $5.1 \mathrm{~nm}$ 的层状结 构是源自于两段簇合物的近似球形的几何特征. 本研究 工作的重要意义在于, 以业已存在的两种无机纳米簇为 构筑单元，构筑了簇一族杂化分子, 并实现了尺度约为 5 $\mathrm{nm}$ 的微相分离结构, 为构筑新型的杂化分子并实现超 分子的自组装结构的调控提出了一个新的思路, 也为这 种精细超分子结构的应用提出了新的课题.

\section{4 实验部分}

\section{1 仪器与试剂}

${ }^{1} \mathrm{H}$ NMR,,${ }^{13} \mathrm{C}$ NMR, ${ }^{31} \mathrm{P}$ NMR 和 ${ }^{29} \mathrm{Si}$ NMR 在氛代溶 剂 $\left(\mathrm{CDCl}_{3}, \mathrm{CD}_{3} \mathrm{CN}, \mathrm{DMSO}-d_{6}\right)$ 中使用 Bruker AVANCE III $400 \mathrm{MHz}$ 核磁谱仪记录数据. 红外光谱采用美国 Bio-Rad 公司 FTS 6000 光谱仪, $\mathrm{KBr}$ 稀释漫反射模式测 试. 电喷雾电离质谱(ESI-MS)采用美国 Thermo Fisher Scientific 公司 Finnigan LCQ Advantage 记录数据. X 射 线粉末衍射(XRD)采用日本理学公司 $\mathrm{D} / \mathrm{max}-2500$ 进行 测试, 发射器为 $\mathrm{CuK \alpha}(\lambda=0.154 \mathrm{~nm})$, 扫描范围为 $0.7^{\circ} \sim 40^{\circ}$. 场发射透射电子显微镜(TEM)采用荷兰 FEI 公司 Tecnai G2 F20 进行测试, 装备能量色散 X 射线光 谱探头(EDX), 加速电压为 $200 \mathrm{kV}$.

Wells-Dawson 型多金属氧簇 $\left(\mathrm{K}_{10} \mathrm{P}_{2} \mathrm{~W}_{17} \mathrm{O}_{61} \cdot 10 \mathrm{H}_{2} \mathrm{O}\right)$ 按照文献[29]方法合成; 1-氨基丙基-3,5,7,9,11,13,15-七 异丁基倍半硅氧烷购自于 Hybrid Plastics 公司; 戊炔酸 购自于 Sigma-Aldrich 公司; 氯甲基苯基三甲氧基硅烷、 1-(3-二甲氨基丙基)-3-乙基碳二亚胺(EDC)和 4-二甲氨 基吡啶(DMAP)购自于 Alfa Aesar 公司; 其它试剂及溶 剂购自于常规试剂公司.

\section{2 炔基倍半硅氧烷(POSS-yne, 2)的制备}

称取伐炔酸 $0.11 \mathrm{~g}$ (1.14 mmol, 1 equiv.), EDC 0.33 $\mathrm{g}$ (1.71 mmol, 1.5 equiv.), DMAP $0.03 \mathrm{~g}(0.23 \mathrm{mmol}, 0.2$ equiv.)溶于 $25 \mathrm{~mL} \mathrm{CH}_{2} \mathrm{Cl}_{2}$ 中, 在氩气保护下, 冰水浴反 应 $2.5 \mathrm{~h}$ 后缓慢滴加 $1 \mathrm{~g}(1.14 \mathrm{mmol})$ 氨基倍半硅氧烷的 $\mathrm{CH}_{2} \mathrm{Cl}_{2}$ 溶液, 室温反应 $24 \mathrm{~h}$ 后, 水洗三次后旋蒸浓缩, 缓慢加入水与乙醇的混合溶剂 $\left(V_{\text {水 }} / V_{\text {乙碚 }}=1: 4\right)$, 得到白 色沉淀, 过滤、干燥得粗产物, 粗产物经硅胶色谱纯化, 淋洗液为乙酸乙酯/石油醚 $(4: 15)$, 得白色固体 $0.92 \mathrm{~g}$. 产率为 $84 \%$. 产物通过 ${ }^{1} \mathrm{H}$ NMR, ${ }^{13} \mathrm{C}$ NMR, ESI-MS 和 IR 表征方法确定了其化学结构, 数据见支持信息. ${ }^{1} \mathrm{H}$ 
NMR (400 MHz, $\left.\mathrm{CDCl}_{3}\right) \delta: 0.60(\mathrm{~d}, J=6.9 \mathrm{~Hz}, 16 \mathrm{H}$, $\left.\mathrm{CH}_{2} \mathrm{Si}\right), 0.95\left(\mathrm{~d}, J=6.6 \mathrm{~Hz}, 42 \mathrm{H}, \mathrm{CH}_{3}\right), 1.57 \sim 1.62(\mathrm{~m}$, $\left.2 \mathrm{H}, \mathrm{CH}_{2} \mathrm{CH}_{2} \mathrm{Si}\right), 1.80 \sim 1.90\left(\mathrm{~m}, 7 \mathrm{H}, \mathrm{CH}\left(\mathrm{CH}_{3}\right)_{2}\right), 1.99$ (t, $\left.J=2.41 \mathrm{~Hz}, 1 \mathrm{H}, \mathrm{CH}_{2} \mathrm{CCH}\right), 2.38$ (t, $J=7.2 \mathrm{~Hz}, 2 \mathrm{H}$, $\left.\mathrm{CH}_{2} \mathrm{CCH}\right), 2.51 \sim 2.55\left(\mathrm{~m}, 2 \mathrm{H}, \mathrm{C}(\mathrm{O}) \mathrm{CH}_{2}\right), 3.25 \sim 3.30(\mathrm{~m}$, $\left.2 \mathrm{H}, \mathrm{NHCH}_{2}\right), 5.60(\mathrm{~s}, 1 \mathrm{H}, \mathrm{NH}) ;{ }^{13} \mathrm{C} \mathrm{NMR}(100 \mathrm{MHz}$, $\left.\mathrm{CDCl}_{3}\right) \delta: 9.44,14.95,22.48,22.97,23.82,25.65,35.48$, 41.80, 69.24, 83.02, 170.66; IR (KBr) v: 3312 (m), 2957 (s), 2877 (s), $2121(\mathrm{w}), 1647$ (m), 1550 (w), 1465 (m), 1230 (s), 1110 (vs), $744(\mathrm{~m}), 482(\mathrm{~m}) \mathrm{cm}^{-1}$. ESI-MS (positive mode, $\mathrm{CH}_{2} \mathrm{Cl}_{2}$ ) $\mathrm{m} / \mathrm{z}$ : 954.3, 976.2, 1030.6.

\section{3 叠氮修饰的多金属氧簇 $\left(\mathrm{POM}-2 \mathrm{~N}_{3}, 4\right)$ 的制备}

量取 $355 \mu \mathrm{L}$ 氯甲基苯基三甲氧基硅烷溶于 $60 \mathrm{~mL}$ 水与乙腈混合溶剂 $\left[V\left(\mathrm{H}_{2} \mathrm{O}\right) / V\left(\mathrm{CH}_{3} \mathrm{CN}\right)=2: 1\right]$ 中, 加入 $1.96 \mathrm{~g} \mathrm{~K}_{10} \mathrm{P}_{2} \mathrm{~W}_{17} \mathrm{O}_{61} \bullet 10 \mathrm{H}_{2} \mathrm{O}$, 此时反应体系为白色乳浊 液, 随之用 $1 \mathrm{~mol} / \mathrm{L}$ 的盐酸调节反应体系的酸性, 使其 $\mathrm{pH} \approx 2$, 此时反应为绿色澄清溶液, 室温下反应 $6.5 \mathrm{~h}$ 后, 旋蒸出大部分的 $\mathrm{CH}_{3} \mathrm{CN}$ 溶剂, 将所得到的浑浊液滴加 到 $10 \mathrm{~mL}, 0.20 \mathrm{~g} / \mathrm{mL}$ 的 $\mathrm{NBu}_{4} \mathrm{Br}$ 的水溶液中进行沉淀处 理, $10 \mathrm{~min}$ 后将得到的沉淀抽滤, 滤饼依次用水、乙醇、 乙醚洗涤, 干燥, 得白色固体 $1.96 \mathrm{~g}$, 产率为 $80 \%$. 再称 取 $1.00 \mathrm{~g}$ 所得样品溶解在 $35 \mathrm{~mL}$ 的 DMF 溶剂中, 加入 $0.10 \mathrm{~g} \mathrm{NaN}_{3}$, 随后补加少量水, 使加入的 $\mathrm{NaN}_{3}$ 全部溶 解, 在 $50{ }^{\circ} \mathrm{C}$ 下反应 $24 \mathrm{~h}$. 反应结束后旋出大部分溶剂, 然后加入 $20 \mathrm{~mL} 0.20 \mathrm{~g} / \mathrm{mL}$ 的 $\mathrm{NBu}_{4} \mathrm{Br}$ 水溶液, 数分钟 后，抽滤所得到的沉淀，依次用水、乙醇、乙醚洗涤，干 燥, 得白色固体 $0.90 \mathrm{~g}$, 产率为 $88 \%$, 详细表征数据见 支持信息. ${ }^{1} \mathrm{H}$ NMR $\left(400 \mathrm{MHz}, \mathrm{DMSO}-d_{6}\right) \delta: 0.94(\mathrm{t}, J=$ $\left.7.3 \mathrm{~Hz}, 72 \mathrm{H}, \mathrm{CH}_{3}\right), 1.28 \sim 1.37\left(\mathrm{~m}, 48 \mathrm{H}, \mathrm{CH}_{2} \mathrm{CH}_{3}\right), 1.54 \sim$ $1.61\left(\mathrm{~m}, 48 \mathrm{H}, \mathrm{CH}_{2} \mathrm{CH}_{2} \mathrm{CH}_{3}\right), 3.15 \sim 3.19(\mathrm{~m}, 48 \mathrm{H}$, $\left.\mathrm{CH}_{2} \mathrm{CH}_{2} \mathrm{CH}_{2} \mathrm{CH}_{3}\right), 4.45$ (s, 4H, $\left.\mathrm{CH}_{2} \mathrm{~N}_{3}\right), 7.36$ (d, $J=7.5$ $\left.\mathrm{Hz}, \quad 4 \mathrm{H}, \quad \mathrm{SiC}(\mathrm{CH})_{2}\right), 7.77(\mathrm{~d}, J=7.5 \mathrm{~Hz}, 4 \mathrm{H}$, $\left.(\mathrm{CH})_{2} \mathrm{CCH}_{2} \mathrm{~N}_{3}\right) ;{ }^{31} \mathrm{P}$ NMR (162 MHz, DMSO- $\left.d_{6}\right) \delta$ : $-10.35\left(\mathrm{~s}, \mathrm{PW}_{8}\right),-13.36\left(\mathrm{~s}, \mathrm{PW}_{9}\right)$; IR (KBr) v: 2961 (m), 2872 (m), $2097(\mathrm{~m}), 1487$ (m), 1602 (w), 1088 (s), 1040 (s), 813 (vs) cm ${ }^{-1}$. ESI-MS (negative mode, $\mathrm{CH}_{3} \mathrm{CN}$ ) $\mathrm{m} / z$ : 1125.6, 1184.6, 1500.7, 1582.1, 1661.5, 1741.8 .

\section{4 多金属氧簇一倍半硅氧烷簇杂化分子 (POM- 2POSS, 5)的制备}

称取 POM-N $\mathrm{P}_{3}$ (0.28 g, 1 equiv.), POSS-yne (0.14 g, 3 equiv.)溶于 $30 \mathrm{~mL} \mathrm{DMF}$ 中, 冷冻除氧 $30 \mathrm{~min}$ 后, 随之加 入 $\mathrm{CuSO}_{4} \cdot 5 \mathrm{H}_{2} \mathrm{O}$ (25.94 mg, 2.2 equiv.)和抗坏血酸(89.10 $\mathrm{mg}, 9$ equiv.), 在 $50{ }^{\circ} \mathrm{C}$ 下反应 $5 \mathrm{~d}$. 反应结束后, 旋蒸浓 缩后滴加到溴化四丁基铵的甲醇溶液中, 干燥后溶于少 量乙腈, 除去不溶固体, 滤液旋干后得白色固体 $0.11 \mathrm{~g}$, 产率为 $32 \%$, 详细表征数据见支持信息. ${ }^{1} \mathrm{H}$ NMR (400 $\left.\mathrm{MHz}, \mathrm{CD}_{3} \mathrm{CN}\right) \delta: 0.61 \sim 0.64\left(\mathrm{~m}, 32 \mathrm{H}, \mathrm{CH}_{2} \mathrm{Si}\right), 0.96 \sim 1.01$ $\left(\mathrm{m}, 156 \mathrm{H},-\mathrm{CH}_{3}\right), 1.36 \sim 1.45\left(\mathrm{~m}, 52 \mathrm{H} ; \mathrm{CH}_{2} \mathrm{CH}_{2} \mathrm{Si}\right.$,
$\left.\mathrm{CH}_{2} \mathrm{CH}_{3}\right), 1.60 \sim 1.68\left(\mathrm{~m}, 48 \mathrm{H}, \mathrm{NCH}_{2} \mathrm{CH}_{2}\right), 1.83 \sim 1.90$ $\left(\mathrm{m}, 14 \mathrm{H}, \mathrm{CH}_{2} \mathrm{CH}\left(\mathrm{CH}_{3}\right)_{2}\right), 2.45(\mathrm{t}, J=6.77 \mathrm{~Hz}, 4 \mathrm{H}$, $\left.\mathrm{COCH}_{2}\right), 2.88 \sim 2.91\left(\mathrm{~m}, 4 \mathrm{H}, \mathrm{COCH}_{2} \mathrm{CH}_{2}\right), 3.12 \sim 3.14(\mathrm{~m}$, $\left.52 \mathrm{H}, \mathrm{NCH}_{2}, \mathrm{NHCH}_{2}\right), 5.50\left(\mathrm{~s}, 4 \mathrm{H}, \mathrm{PhCH}_{2}\right), 6.46(\mathrm{~s}, 2 \mathrm{H}$, $\mathrm{NH}), 7.29\left(\mathrm{~d}, J=7.43 \mathrm{~Hz}, 4 \mathrm{H}, \mathrm{NNCH}_{2} \mathrm{C}(\mathrm{CHCH})_{2}\right), 7.54$ (s, 2H, NNCH), 7.82 (d, $\left.J=7.22 \mathrm{~Hz}, 4 \mathrm{H}, \mathrm{NNCH}_{2} \mathrm{C}(\mathrm{CH})_{2}\right)$; ${ }^{31} \mathrm{P} \mathrm{NMR}\left(162 \mathrm{MHz}, \mathrm{CDCl}_{3}\right) \delta:-10.35\left(\mathrm{~s}, \mathrm{PW}_{8}\right)$, $-13.36\left(\mathrm{~s}, \mathrm{PW}_{9}\right) ;{ }^{29} \mathrm{Si} \mathrm{NMR}\left(79.4 \mathrm{MHz}, \mathrm{CDCl}_{3}\right) \delta$ : -66.56, -67.65, - 67.91; ${ }^{13} \mathrm{C}$ NMR (100 MHz, $\left.\mathrm{CDCl}_{3}\right)$ $\delta: 9.60,14.04,19.74,22.52,23.00,23.87,24.17,25.73$, 29.73, 31.96, 42.01, 53.26, 58.36, 122.02, 126.89, 135.64, 136.84, 141.97, 146.72, 172.07; IR (KBr) v: 3337 (w), 2956 (s), 2871 (s), 1793 (w), 1664 (m), 1108 (vs), 954 (s), 917 (s), 813 (vs) $\mathrm{cm}^{-1}$. ESI-MS (negative mode, $\mathrm{CH}_{2} \mathrm{Cl}_{2}$ ) $m / z: 1603.4,1662.6$. Anal. calcd for $\left[(n-\mathrm{Bu})_{4} \mathrm{~N}\right]_{6}{ }^{-}$ $\left[\left(\mathrm{P}_{2} \mathrm{~W}_{17} \mathrm{O}_{61}\right)\left[\mathrm{SiC}_{6} \mathrm{H}_{4} \mathrm{CH}_{2}\left(\mathrm{C}_{2} \mathrm{HN}_{3}\right) \mathrm{CH}_{2} \mathrm{CH}_{2} \mathrm{CONH}\left(\mathrm{C}_{31} \mathrm{H}_{69^{-}}\right.\right.\right.$ $\left.\left.\left.\mathrm{Si}_{8} \mathrm{O}_{12}\right)\right]_{2} \mathrm{O}\right]$ : C 27.80, H 4.85, N 2.49; found C 27.92, $\mathrm{H}$ $4.82, \mathrm{~N} 2.45$.

\section{References}

[1] Gómez-Romero, P.; Sanchez, C. Functional Hybrid Materials, Wiley-VCH, Weinheim, 2006.

[2] Kickelbick, G. Hybrid Materials: Synthesis, Characterization, and Applications, Wiley-VCH, Weinheim, 2007.

[3] Clément, S.; Kenneth, J. S.; Susumu, K. Chem. Soc. Rev. 2011, 40, 471.

[4] Sanchez, C.; Julián, B.; Belleville, P.; Popall, M. J. Mater. Chem. $\mathbf{2 0 0 5}, 15,3559$.

[5] Zhang, Z. L.; Horsch, M. A.; Lamm, M. H.; Glotzer, S. C. Nano Lett. 2003, 3, 1341.

[6] Glotzer, S. C.; Horsch, M. A.; Iacovella, C. R.; Zhang, Z. L.; Chan, E. R.; Zhang, X. Curr. Opin. Colloid Interface Sci. 2005, 10, 287.

[7] Šarić, A.; Bozorgui, B.; Cacciuto, A. J. Phys. Chem. B 2011, 115 7182.

[8] Filion, L.; Marechal, M.; Oorschot, B. V.; Pelt, D.; Smallenburg, F.; Dijkstra, M. Phys. Rev. Lett. 2009, 103, 188302.

[9] Pradeep, C. P.; Misdrahi, M. F.; Li, F. Y.; Zhang, J.; Xu, L.; Long, D. L.; Liu, T. B.; Cronin, L. Angew. Chem., Int. Ed. 2009, 48, 8309.

[10] Han, Y. K.; Xiao, Y.; Zhang, Z. J.; Liu, B.; Zheng, P.; He, S. J.; Wang, W. Macromolecules 2009, 42, 6543.

[11] Hu, M. B.; Xia, N.; Yu, W.; Ma, C.; Tang, J.; Hou, Z. Y.; Zheng, P.; Wang, W. Polym. Chem. 2012, 3, 617.

[12] Landsmann, S.; Lizandara-Pueyo, C.; Polarz, S. J. Am. Chem. Soc. 2010, 132, 5315.

[13] Landsmann, S.; Wessig, M.; Schmid, M.; Cölfen, H.; Polarz, S Angew. Chem., Int. Ed. 2012, 51, 5995.

[14] Hu, M. B.; Hou, Z. Y.; Xiao, Y.; Yu, W.; Ma, C.; Ren, L. J.; Zheng, P.; Wang, W. Langmuir 2013, 29, 5714

[15] Wang, X. L.; Wang, Y. L.; Miao, W. K.; Hu, M. B.; Tang, J.; Yu, W.; Hou, Z. Y.; Zheng, P.; Wang, W. Langmuir 2013, $29,6537$.

[16] Yu, X. F.; Zhong, S.; Li, X. P.; Tu, Y. F.; Yang, S. G.; Van Horn, R. M.; Ni, C. Y.; Pochan, D. J.; Quirk, R. P.; Wesdemiotis, C.; Zhang, W. B.; Cheng, S. Z. D. J. Am. Chem. Soc. 2010, 132, 16741.

[17] Li, Y.; Zhang, W. B.; Hsieh, I.; Zhang, G. L.; Cao, Y.; Li, X. P.; Wesdemiotis, C.; Lotz, B.; Xiong, H. M.; Cheng, S. Z. D. J. Am. Chem. Soc. 2011, 133, 10712.

[18] Sun, H. J.; Tu, Y. F.; Wang, C. L.; Van Horn, R. M.; Tsai, C. C.; Graham, M. J.; Sun, B.; Lotz, B.; Zhang, W. B.; Cheng, S. Z. D. J. Mater. Chem. 2011, 21, 14240.

[19] Yu, X. F.; Zhang, W. B.; Yue, K.; Li, X. P.; Liu, H.; Xin, Y.; Wang, C. L.; Wesdemiotis, C.; Cheng, S. Z. D. J. Am. Chem. Soc. 2012, 134,7780 .

[20] Polyoxometalate Chemistry: From Topology via Self-Assembly to Applications, Eds.: Pope, M. T.; Müller, A., Springer, Netherlands, 
2001.

[21] Special thematic issue on polyoxometalates: Chem. Rev. 1998, 98, $1-388$.

[22] Proust, A.; Thouvenot, R.; Gouzerh, P. Chem. Commun. 2008, 1837.

[23] Dolbecq, A.; Dumas, E.; Mayer, C. R.; Mialane, P. Chem. Rev. 2010, 110, 6009 .

[24] Proust, A.; Matt, B.; Villanneau, R.; Guillemot, G.; Gouzerh, P.; Izzet, G. Chem. Soc. Rev. 2012, 41, 7605.

[25] Marcella, B.; Mauro, C.; Gianfranco, S.; Alessandro, B. Adv. Synth. Catal. 2004, 346, 648.

[26] Cordes, D. B.; Lickiss, P. D.; Rataboul, F. Chem. Rev. 2010, 110,
2081.

[27] Tanaka, K.; Chujo, Y.J. Mater. Chem. 2012, 22, 1733.

[28] Odobel, F.; Severac, M.; Pellegrin, Y.; Blart, E.; Fosse, C.; Cannizzo, C.; Mayer, C. R.; Elliott, K. J.; Harriman, A. Chem. Eur. J. 2009, 15, 3130 .

[29] Contant, R.; Klemperer, W. G.; Yaghi, O., In Inorganic Syntheses, Mc Graw-Hill, New York, 1990, Vol. 27, p. 104

[30] Kolb, H. C.; Finn, M. G.; Sharpless, K. B. Angew. Chem., Int. Ed. 2001, 40, 2004.

[31] Hamley, I. W. The Physics of Block Copolymers, Oxford University Press, Oxford, 1999. 